\title{
One-loop fluctuations of semi-local self-dual vortices
}

\author{
A. Alonso Izquierdo ${ }^{(a)}$, W. Garcia Fuertes ${ }^{(b)}$ \\ M. de la Torre Mayado ${ }^{(c)}$, J. Mateos Guilarte ${ }^{(c)}$ \\ (a) Departamento de Matematica Aplicada and IUFFyM, Universidad de Salamanca, SPAIN \\ (b) Departamento de Fisica, Universidad de Oviedo, SPAIN \\ (c) Departamento de Fisica and IUFFyM, Universidad de Salamanca, SPAIN
}

\begin{abstract}
Mass shifts induced by one-loop fluctuations of semi-local self-dual vortices are computed. The procedure is based on canonical quantization and heat kernel/zeta function regularization methods. The issue of the survival of the classical degeneracy in the semi-classical regime is explored.
\end{abstract}

\section{Introduction}

In this communication we shall deal with one-loop mass shifts for the semilocal self-dual topological solitons -SSTS in the sequel- that arise in the (2+1)-dimensional semilocal Abelian Higgs model; see [1] for a review of the history and properties of these classical solitonic backgrounds. On the analytical side, a formula will be derived that involves the coefficients of the heat-kernel expansion associated with the second-order fluctuation operator. Additionally, numerical methods are used to generate the solutions and to compute the coefficients. All this together will allow us to obtain numerical results for one-loop SSTS mass shifts.

Control of the ultra-violet divergences arising in the procedure is achieved by using heat kernel/zeta function regularization methods. In the absence of detailed knowledge of the spectrum of the differential operator governing second-order fluctuations around vortices, the expansion of the associated heat kernel will be used in a way akin to that developed in the computation of one-loop mass shifts for one-dimensional kinks; see [3. In fact, a similar technique has been applied previously to compute the mass shift for the supersymmetric kink [4, although in this latter case the boundary conditions must respect supersymmetry. In the case of vortices, the only available results refer to either supersymmetric vortices, achieved by Vassilevich and the Stony Brook/Wien group, [5], 6], or non-supersymmetric self-dual vortices, obtained by our group, [7.

The closely related issue of computing the quantum energy of QED flux tubes due to fermionic fluctuations has been addressed in [10] and, more recently, in the papers [11 and 12. Quantum energies of the more subtle electroweak strings caused by fermionic fluctuations have been thoroughly studied in 13 from a $(2+1)$-dimensional point of view for $\theta=0$ Weinberg angle. We shall concentrate on the value $\theta=\frac{\pi}{2}$. For this weak mixing angle the $S U(2)$ gauge field decouples, the strings become topologically stable, and a broader class of topological solitons arise because the Higgs vacuum manifold becomes the $S^{3}$-sphere Hopf bundle. We shall restrict ourselves, however, to consider only the bosonic fluctuations over topological solitons saturating the Bogomolny bound. This is in contrast to the work mentioned above where fermionic fluctuations dominate because the fermions carry a high enough number of colors.

The study of quantum fluctuations of topological defects arising in models that describe sub-atomic phenomena is a very important and difficult subject. With the exceptions of sine-Gordon and $\lambda(\phi)_{2}^{4}$ kinks, the knowledge of the spectrum of the second-order differential operators governing these fluctuations is non complete. Therefore, asymptotic methods, phase shifts, high-temperature expansions, etcetera, must be 
used. In particular, one must compute the $L^{2}$ trace of the square root of a second-order differential operator, a problem for which the zeta function/heat kernel regularization techniques, see [14, are specially suitable. Unfortunately, difficulties with this procedure increase with the dimension of space-time. Nevertheless, the experience with these planar examples makes conceivable the possibility of computing the one-loop mass shift for BPS magnetic monopoles sometime in the future.

\section{The planar semi-local Abelian Higgs model}

We write the action governing the dynamics of the semi-local AHM in the form:

$$
S=\frac{v}{e} \int d^{3} x\left[-\frac{1}{4} F_{\mu \nu} F^{\mu \nu}+\frac{1}{2}\left(D_{\mu} \Phi\right)^{*} D^{\mu} \Phi-\frac{\kappa^{2}}{8}\left(\Phi^{\dagger} \Phi-1\right)^{2}\right] \quad .
$$

Besides the Abelian gauge field $A_{\mu}\left(x^{\mu}\right)$, there is a doublet of complex scalar fields. The action is invariant with respect to $U(1)$ gauge (local) and global (rigid) $S U(2)$ transformations, and it is no more than the bosonic sector of the electro-weak theory when the weak mixing angle is $\frac{\pi}{2}$. Note that we define the electric charge unconventionally: $Q=-T_{3}+\frac{1}{2} Y$, in such a way that the neutral scalar field is the upper component of the weak iso-spinorial Higgs field.

A shift of the complex scalar field from the vacuum

$$
\Phi\left(x^{\mu}\right)=\left(\begin{array}{c}
\Phi_{1}\left(x^{\mu}\right) \\
\Phi_{2}\left(x^{\mu}\right)
\end{array}\right)=\left(\begin{array}{c}
\Phi_{1}^{1}\left(x^{\mu}\right)+i \Phi_{1}^{2}\left(x^{\mu}\right) \\
\Phi_{2}^{1}\left(x^{\mu}\right)+i \Phi_{2}^{2}\left(x^{\mu}\right)
\end{array}\right)=\left(\begin{array}{c}
1+H\left(x^{\mu}\right)+i G\left(x^{\mu}\right) \\
\sqrt{2} \varphi\left(x^{\mu}\right)
\end{array}\right)
$$

and choice of the Feynman-'t Hooft renormalizable gauge $R\left(A_{\mu}, G\right)=\partial_{\mu} A^{\mu}\left(x^{\mu}\right)-G\left(x^{\mu}\right)$ lead us to write the action in terms of Higgs $H$, real Goldstone $G$, complex Goldstone $\varphi$, vector boson $A_{\mu}$ and ghost $\chi$ fields:

$$
\begin{aligned}
S+S_{\text {g.f. }}+S_{\text {ghost }} & =\frac{v}{e} \int d^{3} x\left[-\frac{1}{2} A_{\mu}\left[-g^{\mu \nu}(\square+1)\right] A_{\nu}+\partial_{\mu} \chi^{*} \partial^{\mu} \chi-\chi^{*} \chi\right. \\
& +\frac{1}{2} \partial_{\mu} G \partial^{\mu} G-\frac{1}{2} G^{2}+\frac{1}{2} \partial_{\mu} H \partial^{\mu} H-\frac{\kappa^{2}}{2} H^{2}+\partial_{\mu} \varphi^{*} \partial^{\mu} \varphi \\
& -\frac{\kappa^{2}}{2} H\left(H^{2}+G^{2}\right)+A_{\mu}\left(\partial^{\mu} H G-\partial^{\mu} G H\right)+H\left(A_{\mu} A^{\mu}-\chi^{*} \chi\right)+i A_{\mu}\left(\varphi^{*} \partial^{\mu} \varphi-\varphi \partial^{\mu} \varphi^{*}\right) \\
& \left.+A_{\mu} A^{\mu}|\varphi|^{2}-\frac{\kappa^{2}}{8}\left(H^{2}+G^{2}\right)^{2}+\frac{1}{2}\left(G^{2}+H^{2}\right) A_{\mu} A^{\mu}-\frac{\kappa^{2}}{2}|\varphi|^{2}\left(|\varphi|^{2}+H^{2}+G^{2}+2 H\right)\right]
\end{aligned}
$$

\section{$2.1 \quad$ Vacuum energy}

Canonical quantization promoting the coefficients of the plane wave expansion around the vacuum of the fields to operators provides the free quantum Hamiltonian. Besides the plane wave expansions in a normalizing plate of very huge area $L^{2}$ of the fields of the Abelian Higgs model considered in the third paper of Reference [7] we must also take into account the massless complex Goldstone bosons:

- If $m=e v$,

$$
\begin{gathered}
\delta \varphi\left(x_{0}, \vec{x}\right)=\frac{e}{m L} \sqrt{\frac{\hbar}{m}} \sum_{\vec{k}} \frac{1}{\sqrt{2 \gamma(\vec{k})}}\left[f^{*}(\vec{k}) e^{i k x}+g(\vec{k}) e^{-i k x}\right] \quad, \quad \gamma(\vec{k})=+\sqrt{\vec{k} \vec{k}} \\
{\left[\hat{f}(\vec{k}), \hat{f}^{\dagger}(\vec{q})\right]=\left[\hat{g}(\vec{k}), \hat{g}^{\dagger}(\vec{q})\right]=\delta_{\vec{k} \vec{q}} \Rightarrow H^{(2)}[\delta \hat{\varphi}]=\hbar m \sum_{\vec{k}} \gamma(\vec{k})\left(\hat{f}^{\dagger}(\vec{k}) \hat{f}(\vec{k})+\hat{g}^{\dagger}(\vec{k}) \hat{g}(\vec{k})+1\right) .}
\end{gathered}
$$

\footnotetext{
${ }^{1}$ Details of our conventions and calculations are given in 8
} 
The vacuum energy is the sum of five contributions: if $\triangle=\sum_{j=1}^{2} \frac{\partial}{\partial x_{j}} \cdot \frac{\partial}{\partial x_{j}}$ denotes the Laplacian,

$$
\begin{gathered}
\Delta E_{0}^{(1)}=\sum_{\vec{k}} \sum_{\alpha} \frac{\hbar m}{2} \omega(\vec{k})=\frac{3 \hbar m}{2} \operatorname{Tr}[-\Delta+1]^{\frac{1}{2}}, \Delta E_{0}^{(2)}=\sum_{\vec{k}} \frac{\hbar m}{2} \nu(\vec{k})=\frac{\hbar m}{2} \operatorname{Tr}\left[-\Delta+\kappa^{2}\right]^{\frac{1}{2}} \\
\Delta E_{0}^{(3)}=\sum_{\vec{k}} \frac{\hbar m}{2} \omega(\vec{k})=\frac{\hbar m}{2} \operatorname{Tr}[-\triangle+1]^{\frac{1}{2}}, \quad \Delta E_{0}^{(4)}=\sum_{\vec{k}} \hbar m \gamma(\vec{k})=\hbar m \operatorname{Tr}[-\triangle]^{\frac{1}{2}} \\
E_{0}^{(5)}=-\sum_{\vec{k}} \hbar m \omega(\vec{k})=-\hbar m \operatorname{Tr}[-\triangle+1]^{\frac{1}{2}}
\end{gathered}
$$

come from the vacuum fluctuations of the vector boson, Higgs, real Goldstone, complex Goldstone, and ghost fields. Ghost fluctuations, however, cancel the contribution of temporal vector bosons and real Golstone particles, and the vacuum energy in the planar semi-local AHM is due only to Higgs particles, complex Goldstone bosons, and transverse massive vector bosons:

$$
\Delta E_{0}=\sum_{r=1}^{5} \Delta E_{0}^{(r)}=\hbar m \operatorname{Tr}[-\triangle+1]^{\frac{1}{2}}+\frac{\hbar m}{2} \operatorname{Tr}\left[-\triangle+\kappa^{2}\right]^{\frac{1}{2}}+\hbar m \operatorname{Tr}[-\triangle]^{\frac{1}{2}}
$$

\subsection{Semi-local self-dual topological solitons}

At the critical point between Type I and Type II superconductivity, $\kappa^{2}=1$, the energy can be arranged in a Bogomolny splitting:

$$
E=\frac{m^{2}}{2 e^{2}} \int d^{2} x\left(\left\|D_{1} \Phi \pm i D_{2} \Phi\right\|^{2}+\left[F_{12} \pm \frac{1}{2}\left(\Phi^{\dagger} \Phi-1\right)\right]^{2}\right)+\frac{m^{2}}{2} \frac{|g|}{e^{2}} \quad, \quad g=\int d^{2} x F_{12}=2 \pi l, l \in \mathbb{Z} .
$$

Therefore, the solutions of the first-order equations $D_{1} \Phi \pm i D_{2} \Phi=0=F_{12} \pm \frac{1}{2}\left(\Phi^{\dagger} \Phi-1\right)$ are absolute minima of the energy, hence stable, in each topological sector with a classical mass proportional to the magnetic flux. It has been shown in 22 that there is a $4 l$-dimensional moduli space of such solitonic solutions interpolating between the Nielsen-Olesen -NO in the sequel- vortices of the Abelian Higgs model and the $\mathbb{C} P^{1}$-lumps of the planar non-linear sigma model.

Assuming a purely vorticial vector field plus the spherically symmetric ansatz

$$
\begin{aligned}
\phi_{1}\left(x_{1}, x_{2}\right)=f(r) \cos l \theta & , & \phi_{2}\left(x_{1}, x_{2}\right) & =f(r) \sin l \theta \\
\phi_{3}\left(x_{1}, x_{2}\right)=h(r) \cos (\lambda+n \theta) & , & \phi_{4}\left(x_{1}, x_{2}\right) & =h(r) \sin (\lambda+n \theta) \quad, \quad \lambda \in \mathbb{C}, n \in \mathbb{Z} \\
A_{1}\left(x_{1}, x_{2}\right)=-l \frac{\alpha(r)}{r} \sin \theta & , & A_{2}\left(x_{1}, x_{2}\right) & =l \frac{\alpha(r)}{r} \cos \theta \quad,
\end{aligned}
$$

$g=-\oint_{r=\infty} d x_{i} A_{i}=-l \oint_{r=\infty} \frac{\left[x_{2} d x_{1}-x_{1} d x_{2}\right]}{r^{2}}=2 \pi l$, the first-order equations reduce t 2$]$

$$
\frac{1}{r} \frac{d \alpha}{d r}(r)=\mp \frac{1}{2 l}\left(f^{2}(r)+h^{2}(r)-1\right) \quad, \quad \frac{d f}{d r}(r)= \pm \frac{l}{r} f(r)[1-\alpha(r)] \quad, \quad \frac{d h}{d r}(r)= \pm \frac{l}{r} h(r)\left[\frac{n}{l}-\alpha(r)\right],
$$

to be solved together with the boundary conditions

$$
\begin{array}{lllr}
\lim _{r \rightarrow \infty} f(r)=1 \quad, & \lim _{r \rightarrow \infty} h(r)=0 \quad & \lim _{r \rightarrow \infty} \alpha(r)=1 \\
f(0)=0 & h(0)=h_{0} \delta_{n, 0} & & \alpha(0)=0,
\end{array}
$$

required by energy finiteness plus regularity at the origin (center of the vortex). A partly numerical, partly analytical procedure explained in detail in [8] provides the field profiles $f(r), \alpha(r)$ as well as the magnetic field $B(r)=\frac{l}{2 r} \frac{d \alpha}{d r}$ and the energy density:

$$
\mathcal{E}(r)=\frac{1}{8}\left(\frac{1}{l^{2}}+1\right)\left(1-f^{2}(r)-h(r)^{2}\right)^{2}+\frac{l^{2} f^{2}(r)}{r^{2}}(1-\alpha(r))^{2}+\frac{l^{2} h(r)^{2}}{r^{2}}\left(\frac{|n|}{|l|}-\alpha(r)\right)^{2} \quad .
$$

\footnotetext{
${ }^{2}$ The upper (lower) signs correspond to $l$ and $n$ positive (negative). Finite energy solutions only exists if $|n|<|l|$.
} 
We have worked completely in the last Reference the $l=1, n=0, \lambda=0$ case and plotted the field profiles and the energy density for four values of $h_{0}$. The physical meaning of the parameter $h_{0}$, giving the size and the phase of the $\Phi_{2}$ field for the solution at the origin, is also explained there. We remark that solutions with $h_{0}=0$ are the NO vortices embedded in this system and the growth of $h_{0}$ corresponds to the spread of the energy density of the generic SSTS solutions. Solutions with $\left|h_{0}\right|=1$ are the $C P^{1}$-lumps with energy density homogeneously distributed over the whole plane.

\subsection{Casimir energy of semi-local self-dual topological solitons}

Let us consider small fluctuations around vortices $\Phi\left(x_{0}, \vec{x}\right)=S(\vec{x})+\delta S\left(x_{0}, \vec{x}\right), A_{k}\left(x_{0}, \vec{x}\right)=V_{k}(\vec{x})+$ $\delta a_{k}\left(x_{0}, \vec{x}\right)$, where by $S(\vec{x})$ and $V_{k}(\vec{x})$ we respectively denote the scalar and vector field of the semi-local vortex solutions. Working in the Weyl/background gauge

$$
A_{0}\left(x_{0}, \vec{x}\right)=0 \quad, \quad \partial_{j} \delta a_{j}\left(x_{0}, \vec{x}\right)+\frac{i}{2}\left(S^{\dagger}(\vec{x}) \delta S\left(x_{0}, \vec{x}\right)-\delta S^{\dagger}\left(x_{0}, \vec{x}\right) S(\vec{x})\right)=0 \quad,
$$

the classical energy up to $\mathcal{O}\left(\delta^{2}\right)$ order is:

$$
H^{(2)}+H_{\text {g.f. }}^{(2)}+H_{\text {ghost }}^{(2)}=\frac{v^{2}}{2} \int d^{2} x\left\{\frac{\partial \delta \xi^{T}}{\partial x_{0}} \frac{\partial \delta \xi}{\partial x_{0}}+\delta \xi^{T}\left(x_{0}, \vec{x}\right) K \delta \xi\left(x_{0}, \vec{x}\right)+\delta \chi^{*}(\vec{x}) K^{G} \delta \chi(\vec{x})\right\},
$$

where

$$
\delta \xi\left(x_{0}, \vec{x}\right)=\left(\begin{array}{c}
\delta a_{1}\left(x_{0}, \vec{x}\right) \\
\delta a_{2}\left(x_{0}, \vec{x}\right) \\
\delta S_{1}^{1}\left(x_{0}, \vec{x}\right) \\
\delta S_{1}^{2}\left(x_{0}, \vec{x}\right) \\
\delta S_{2}^{1}\left(x_{0}, \vec{x}\right) \\
\delta S_{2}^{2}\left(x_{0}, \vec{x}\right)
\end{array}\right) \quad, \quad K^{G}=-\triangle+\left|S_{1}(\vec{x})\right|^{2}+\left|S_{2}(\vec{x})\right|^{2}
$$

and

$$
\begin{gathered}
K=\left(\begin{array}{cccccc}
A & 0 & -2 \nabla_{1} S_{1}^{2} & 2 \nabla_{1} S_{1}^{1} & -2 \nabla_{1} S_{2}^{2} & 2 \nabla_{1} S_{2}^{1} \\
0 & A & -2 \nabla_{2} S_{1}^{2} & 2 \nabla_{2} S_{1}^{1} & -2 \nabla_{2} S_{2}^{2} & 2 \nabla_{2} S_{2}^{1} \\
-2 \nabla_{1} S_{1}^{2} & -2 \nabla_{2} S_{1}^{2} & B & -2 V_{k} \partial_{k} & S_{1}^{1} S_{2}^{1}+S_{1}^{2} S_{2}^{2} & S_{1}^{1} S_{2}^{2}-S_{1}^{2} S_{2}^{1} \\
2 \nabla_{1} S_{1}^{1} & 2 \nabla_{2} S_{1}^{1} & 2 V_{k} \partial_{k} & B & -S_{1}^{1} S_{2}^{2}+S_{1}^{2} S_{2}^{1} & S_{1}^{1} S_{2}^{1}+S_{1}^{2} S_{2}^{2} \\
-2 \nabla_{1} S_{2}^{2} & -2 \nabla_{2} S_{2}^{2} & S_{1}^{1} S_{2}^{1}+S_{1}^{2} S_{2}^{2} & -S_{1}^{1} S_{2}^{2}+S_{1}^{2} S_{2}^{1} & C & -2 V_{k} \partial_{k} \\
2 \nabla_{1} S_{2}^{1} & 2 \nabla_{2} S_{2}^{1} & S_{1}^{1} S_{2}^{2}-S_{1}^{2} S_{2}^{1} & S_{1}^{1} S_{2}^{1}+S_{1}^{2} S_{2}^{2} & 2 V_{k} \partial_{k} & C
\end{array}\right) \\
A=-\partial_{k} \partial_{k}+\left|S_{1}\right|^{2}+\left|S_{2}\right|^{2} \quad, \quad B=-\partial_{k} \partial_{k}+\frac{1}{2}\left(3\left|S_{1}\right|^{2}+\left|S_{2}\right|^{2}+2 V_{k} V_{k}-1\right)
\end{gathered}
$$

The general solutions of the linearized field equations

$$
\frac{\partial^{2} \delta \xi_{A}}{\partial x_{0}^{2}}\left(x_{0}, \vec{x}\right)+\sum_{B=1}^{6} K_{A B} \cdot \delta \xi_{B}\left(x_{0}, \vec{x}\right)=0 \quad, \quad K^{G} \delta \chi(\vec{x})=\left(-\triangle+|s(\vec{x})|^{2}\right) \delta \chi(\vec{x})=0
$$

are the eigenfunction expansions (the prime means that zero modes are not included)

$$
\begin{aligned}
\delta \xi_{A}^{\prime}\left(x_{0}, \vec{x}\right) & =\frac{e}{m L} \sqrt{\frac{\hbar}{m}} \cdot \sum_{\vec{k}} \sum_{I=1}^{4} \frac{1}{\sqrt{2 \omega(\vec{k})}}\left[a_{I}^{*}(\vec{k}) e^{i \omega(\vec{k}) x_{0}} u_{A}^{(I) *}(\vec{x} ; \vec{k})+a_{I}(\vec{k}) e^{-i \omega(\vec{k}) x_{0}} u_{A}^{(I)}(\vec{x} ; \vec{k})\right] \\
& +\frac{e}{m L} \sqrt{\frac{\hbar}{m}} \cdot \sum_{\vec{k}} \sum_{I=5}^{6} \frac{1}{\sqrt{2 \gamma(\vec{k})}}\left[a_{I}^{*}(\vec{k}) e^{i \gamma(\vec{k}) x_{0}} u_{A}^{(I) *}(\vec{x} ; \vec{k})+a_{I}(\vec{k}) e^{-i \gamma(\vec{k}) x_{0}} u_{A}^{(I)}(\vec{x} ; \vec{k})\right]
\end{aligned}
$$




$$
\delta \chi^{\prime}\left(x_{0}, \vec{x}\right)=\frac{e}{m L} \sqrt{\frac{\hbar}{m}} \cdot \sum_{\vec{k}} \frac{1}{\sqrt{2 \omega(\vec{k})}}\left[c(\vec{k}) u^{*}(\vec{x} ; \vec{k})+d^{*}(\vec{k}) u(\vec{x} ; \vec{k})\right]
$$

where $A=1,2,3,4,5,6$ and by $u^{(I)}(k), u(k)$ the non-zero eigenfunctions of $K$ and $K^{G}$ are denoted respectively: $I=1,2,3,4, K u^{(I)}(\vec{x})=\omega(\vec{k}) u^{(I)}(\vec{x}), I=5,6, K u^{(I)}(\vec{x})=\gamma(\vec{k}) u^{(I)}(\vec{x}), K^{G} u(\vec{x})=\omega(\vec{k}) u(\vec{x})$. Canonical quantization

$$
\left[\hat{a}_{I}(\vec{k}), \hat{a}_{J}^{\dagger}(\vec{q})\right]=\delta_{I J} \delta_{\vec{k} \vec{q}} \quad, \quad\left\{\hat{c}(\vec{k}), \hat{c}^{\dagger}(\vec{q})\right\}=\delta_{\vec{k} \vec{q}} \quad, \quad\left\{\hat{d}(\vec{k}), \hat{d}^{\dagger}(\vec{q})\right\}=\delta_{\vec{k} \vec{q}}
$$

leads to the quantum free Hamiltonian

$$
\begin{aligned}
\hat{H}^{(2)}+\hat{H}_{\text {g.f. }}^{(2)}+\hat{H}_{\text {Ghost }}^{(2)} & =\hbar m \cdot \sum_{\vec{k}}\left[\sum_{I=1}^{4} \omega(\vec{k})\left(\hat{a}_{I}^{\dagger}(\vec{k}) \hat{a}_{I}(\vec{k})+\frac{1}{2}\right)+\sum_{I=5}^{6} \gamma(\vec{k})\left(\hat{a}_{I}^{\dagger}(\vec{k}) \hat{a}_{I}(\vec{k})+\frac{1}{2}\right)\right] \\
& +\hbar m \cdot \sum_{\vec{k}}\left[\frac{1}{2} \omega(\vec{k})\left(\hat{c}^{\dagger}(\vec{k}) \hat{c}(\vec{k})+\hat{d}^{\dagger}(\vec{k}) \hat{d}(\vec{k})-1\right)\right],
\end{aligned}
$$

and the ground state energy (all the modes non-occupied) of the topological solitons reads:

$$
\triangle E_{\mathrm{TS}}=\frac{\hbar m}{2} \mathrm{STr}^{*} K^{\frac{1}{2}}=\frac{\hbar m}{2} \operatorname{Tr}^{*} K^{\frac{1}{2}}-\frac{\hbar m}{2} \operatorname{Tr}^{*}\left(K^{\mathrm{G}}\right)^{\frac{1}{2}} \quad,
$$

where the star means that zero eigenvalues are not accounted for. Note that the ghost fields are static in this combined Weyl-background gauge and their vacuum energy is one-half with respect to the time-dependent case. Only the Goldstone fluctuations around the vortices must be subtracted. The zero-point vacuum energy renormalization provides the Casimir energy for self-dual $\left(\kappa^{2}=1\right)$ semi-local topological solitons:

$$
\triangle M_{\mathrm{TS}}^{C}=\triangle E_{\mathrm{TS}}-\triangle E_{0}=\frac{\hbar m}{2}\left[\mathrm{STr}^{*} K^{\frac{1}{2}}-\mathrm{S} \operatorname{Tr} K_{0}^{\frac{1}{2}}\right] .
$$

\subsection{Mass renormalization energy}

In $(2+1)$-dimensional model only graphs with one or two external lines are divergent in the vacuum Sector. We choose the following counter-terms to cancel these divergences:

$$
\begin{aligned}
& \mathcal{L}_{\text {c.t. }}^{S}=\frac{\hbar}{2}\left[2\left(\kappa^{2}+1\right) \cdot I(1)+\kappa^{2} \cdot I(0)\right] \cdot\left[\Phi_{1}^{*}\left(x^{\mu}\right) \Phi_{1}\left(x^{\mu}\right)+\Phi_{2}^{*}\left(x^{\mu}\right) \Phi_{2}\left(x^{\mu}\right)-1\right] \\
& \mathcal{L}_{c . t .}^{A}=-\hbar[I(1)+I(0)] \cdot A_{\mu}\left(x^{\mu}\right) A^{\mu}\left(x^{\mu}\right) \quad, \quad I\left(c^{2}\right)=\int \frac{d^{3} k}{(2 \pi)^{3}} \cdot \frac{i}{k^{2}-c^{2}+i \varepsilon}
\end{aligned}
$$

Therefore,

$$
S_{c . t .}=\frac{\hbar}{2} \int d^{3} x\left\{\left[2\left(\kappa^{2}+1\right) \cdot I(1)+\kappa^{2} \cdot I(0)\right] \cdot\left[2 H+H^{2}+G^{2}+2|\varphi|^{2}\right]-2[I(1)+I(0)] \cdot A_{\mu} A^{\mu}\right\}
$$

must be added to the bare action (1) to tame the divergences arising in one-loop order. This specific choice fixes finite renormalizations according to the following criteria:

1. We have used a minimal subtraction scheme taking care only of infinite quantities.

2. By doing this, the choice of scalar field counter-terms sets the no-tadpole condition for the critical value $\kappa^{2}=1$ between Type I and Type II superconductivity, precisely the regime in which we are interested. Vanishing of the tadpole ensures no modification of the $\operatorname{VEV}<\Phi>=(1,0)^{T}$ at one-loop level. This condition is standard in the computation of one-loop mass shifts to supersymmetric and non-supersymmetric kinks and vortices, see [4] and [3]. 
3. Considering no finite counter-terms for the derivative terms of the Higgs, $H$, and Goldstone, $G, \varphi$, fields, as well as their three-valent and four-valent vertices, sets the poles of their masses at their tree levels: $m_{H}=\kappa, m_{G}=1, m_{\varphi}=1$, with residue one.

4. The mass counter-term for the vector boson field plus the no addition of finite counter-terms for derivatives and three- and four-valents vertices of this field keeps also the vector boson mass at its tree level: $m_{A}=1$. Note that a mass term for the $A_{\mu}$ arises already at the tree level in the action (11) as a consequence of the Higgs mechanism in the renormalizable gauge. This point is crucial for staying at the critical value $\kappa^{2}=1$ in the one-loop level.

5. When the zeta function regularization method is used in the computation of one-loop mass shifts to non SUSY and SUSY kinks, the large mass and heat kernel subtraction schemes are known to be equivalent to the vanishing tadpole condition, see [3], 4], and [15. Essentially this means that the no-tadpole condition determines a contribution of the counter-terms to the one-loop kink Hamiltonian energy density which exactly cancels the contribution of the first coefficient of the high-temperature heat function expansion $c_{1}(K)$ to the kink Casimir energy. On the other hand, the contribution to the kink Casimir energy of the zero-order coefficient is exactly canceled by the zero-point vacuum energy renormalization. These two cancelations together ensure that there are no divergences and no quantum corrections in the energy in the infinite mass limit, as it should be: there are no quantum fluctuations of infinite mass.

In the $(2+1)$-dimensional Abelian Higgs model also, only the contributions of $c_{0}(K)$ and $c_{1}(K)$ to the vortex Casimir energies would be non-zero (in fact, infinite) in the infinite mass limit. The contribution of $c_{0}(K)$ is canceled like in the kink case by subtracting the zero-point vacuum energy. The vanishing tadpole condition, however, is necessary but not sufficient to cancel the contribution of $c_{1}(K)$ : one needs also the counter-term to the vector boson mass considered above, see [7].

6. Finally, it would be possible to express all the divergent Feynman amplitudes, up to finite parts, in terms, e.g., of the divergent integral $I(1)$. Our choice of counter-terms, however, respect the global $S U(2)$ symmetry which allows the existence a priori of other topological solitons than the NO vortices.

A detailed calculation of some Feynman amplitudes needed to perform this one-loop renormalization is offered in the last Appendix of Reference [9].

The contribution of these counter-terms to the one-loop mass shift of the SSTS reads:

$$
\Delta M_{\mathrm{TS}}^{R}=\frac{\hbar m}{2} \int d^{2} x\left\{I(1)\left[4\left(1-\left|S_{1}\right|^{2}-\left|S_{2}\right|^{2}\right)-2 V_{k} V_{k}\right]+I(0)\left[\left(1-\left|S_{1}\right|^{2}-\left|S_{2}\right|^{2}\right)-2 V_{k} V_{k}\right]\right\}
$$

and, formally, the total one-loop mass shift is: $\triangle M_{\mathrm{TS}}=\triangle M_{\mathrm{TS}}^{C}+\triangle M_{\mathrm{TS}}^{R}$.

\section{The high-temperature one-loop vortex mass shift formula}

From the high-temperature expansion of the heat kernels

$$
\operatorname{Tr} e^{-\beta K}=\frac{e^{-\beta}}{4 \pi \beta} \cdot \sum_{n=0}^{\infty} \sum_{A=1}^{6} \beta^{n}\left[c_{n}\right]_{A A}(K) \quad, \quad \operatorname{Tr} e^{-\beta K^{\mathrm{G}}}=\frac{e^{-\beta}}{4 \pi \beta} \cdot \sum_{n=0}^{\infty} \beta^{n} c_{n}\left(K^{\mathrm{G}}\right)
$$

the SSTS generalized zeta functions can be written in the form:

$$
\begin{gathered}
\zeta_{K}(s)=\sum_{n=0}^{\infty}\left\{\sum_{A=1}^{4} \frac{\left[c_{n}\right]_{A A}(K)}{4 \pi \Gamma(s)} \cdot \gamma[s+n-1,1]+\sum_{A=5}^{6} \frac{\left[c_{n}(K)\right]_{A A}}{4 \pi \Gamma(s)} \frac{1}{s+n-1}\right\}+\frac{1}{\Gamma(s)} \int_{1}^{\infty} \operatorname{Tr}^{*} e^{-\beta K} d \beta \\
\zeta_{K^{G}}(s)=\sum_{n=0}^{\infty} c_{n}\left(K^{G}\right) \cdot \frac{\gamma[s+n-1,1]}{4 \pi \Gamma(s)}+\frac{1}{\Gamma(s)} \int_{1}^{\infty} d \beta \operatorname{Tr}^{*} e^{-\beta K^{G}} .
\end{gathered}
$$


The diagonal Seeley coefficients $\left[c_{n}\right]_{A A}(K)$ of the K-heat function high-T expansion (resp. the Seeley coefficients $\left.c_{n}\left(K^{G}\right)\right)$ are the integrals over the whole plane of the Seeley densities $\left[c_{n}\right]_{A A}(\vec{x}, \vec{x} ; K)$ which arise in the associated K-heat kernel expansion (resp. the Seeley densities $c_{n}\left(\vec{x}, \vec{x} ; K^{G}\right)$ ):

$$
\left[c_{n}\right]_{A A}(K)=\int d^{2} x\left[c_{n}\right]_{A A}(\vec{x}, \vec{x} ; K) \quad, \quad c_{n}\left(K^{G}\right)=\int d^{2} x c_{n}\left(\vec{x}, \vec{x} ; K^{G}\right)
$$

Neglecting the entire part and setting a large but finite $N_{0}$, the SSTS Casimir energies are regularized as

$$
\begin{aligned}
\Delta M_{\mathrm{TS}}^{C}(s) & =\frac{\hbar \mu}{2}\left(\frac{\mu^{2}}{m^{2}}\right)^{s}\left\{-\frac{4 l}{\Gamma(s)} \int_{0}^{1} d \beta \beta^{s-1}+\sum_{n=1}^{N_{0}}\left[\sum_{A=1}^{4}\left[c_{n}\right]_{A A}(K)-c_{n}\left(K^{G}\right)\right] \cdot \frac{\gamma[s+n-1,1]}{4 \pi \Gamma(s)}+\right. \\
& \left.+\sum_{A=5}^{6} \frac{\left[c_{n}(K)\right]_{A A}}{4 \pi \Gamma(s)} \frac{1}{s+n-1}\right\},
\end{aligned}
$$

where the $4 l$ zero modes have been subtracted: the zero-point vacuum renormalization amounts to ruling out the contribution of the $c_{0}(K)$ and $c_{0}\left(K^{G}\right)$ coefficients. Also, $\Delta M_{\mathrm{TS}}^{R}$ is regularized in a similar way

$$
\Delta M_{\mathrm{TS}}^{R}(s)=\frac{\hbar}{2 \mu L^{2}}\left(\frac{\mu^{2}}{m^{2}}\right)^{s}\left\{\zeta_{-\triangle+1}(s) \cdot \Sigma^{(1)}\left(s(\vec{x}), V_{k}(\vec{x})\right)+\zeta_{-\triangle}(s) \cdot \Sigma^{(0)}\left(s(\vec{x}), V_{k}(\vec{x})\right)\right\}
$$

$\Sigma^{(1)}\left(S, V_{k}\right)=4 \int d^{2} x\left(1-\left|S_{1}\right|^{2}-\left|S_{2}\right|^{2}-\frac{1}{2} V_{k} V_{k}\right) \quad, \quad \Sigma^{(0)}\left(S, V_{k}\right)=\int d^{2} x\left(1-\left|S_{1}\right|^{2}-\left|S_{2}\right|^{2}-2 V_{k} V_{k}\right)$.

The physical limits $s=-\frac{1}{2}$ for $\Delta M_{\mathrm{TS}}^{C}$ and $s=\frac{1}{2}$ for $\Delta M_{\mathrm{TS}}^{R}$ are regular points of the zeta functions. The contribution of the first coefficient of the asymptotic expansion is not compensated by the contribution of the mass renormalization counter-terms:

$$
\begin{aligned}
\Delta M_{T S}^{(1) C}(-1 / 2) & =-\frac{\hbar m}{16 \pi}\left\{\left(\Sigma^{(1)}\left(S, V_{k}\right)+2 \int d^{2} x\left|S_{2}\right|^{2}\left(x_{1}, x_{2}\right)\right) \cdot \frac{\gamma[-1 / 2,1]}{\Gamma(1 / 2)}\right. \\
& \left.-\left(\Sigma^{(0)}\left(S, V_{k}\right)-2 \int d^{2} x\left|S_{2}\right|^{2}\left(x_{1}, x_{2}\right)\right) \cdot \frac{2}{\Gamma(1 / 2)}\right\} \\
\Delta M_{T S}^{R}(1 / 2)= & \frac{\hbar m}{16 \pi} \cdot\left\{\Sigma^{(1)}\left(S, V_{k}\right) \cdot \frac{\gamma[-1 / 2,1]}{\Gamma(1 / 2)}-\frac{2}{\Gamma(1 / 2)} \cdot \Sigma^{(0)}\left(S, V_{k}\right)\right\}
\end{aligned}
$$

Massless particles spoil the large mass subtraction criterion, see [4, and we finally obtain the high-temperature one-loop SSTS mass shift formula:

$$
\begin{aligned}
\Delta M_{T S} & =-\frac{\hbar m}{16 \pi \sqrt{\pi}}\left[\sum_{n=2}^{N_{0}}\left\{\left[\sum_{A=1}^{4}\left[c_{n}(K)\right]_{A A}-c_{n}\left(K^{\mathrm{G}}\right)\right] \cdot \gamma\left[n-\frac{3}{2}, 1\right]+\sum_{A=5}^{6} \frac{\left[c_{n}(K)\right]_{A A}}{n-\frac{3}{2}}\right\}+4 l \cdot 8 \pi\right] \\
& -\frac{\hbar m}{8 \pi \sqrt{\pi}} \cdot \int d^{2} x\left|S_{2}\right|^{2}\left(x_{1}, x_{2}\right) \cdot\left(\gamma\left[-\frac{1}{2}, 1\right]-2\right)
\end{aligned}
$$

\section{Numerical results}

Numerical methods are now implemented in a two-step procedure. First, the Seeley densities are found by means of a symbolic program run in a Mathematica environment on a PC. Second, numerical integration of the Seeley densities on a disk of (non-dimensional) radius $R=10^{4}$ allows us to compute the heat kernel coefficients. We thus find, by setting $N_{0}=6$ and $l=1$, the following numerical results for one-loop mass shifts of semi-local self-dual topological solitons

$$
\begin{array}{ll}
M_{\mathrm{TS}}^{l=1}\left(h_{0}=0.1\right)=m\left(\frac{\pi v}{e}-1.55133 \hbar\right)+o\left(\hbar^{2}\right), & M_{\mathrm{TS}}^{l=1}\left(h_{0}=0.3\right)=m\left(\frac{\pi v}{e}-0.252586 \hbar\right)+o\left(\hbar^{2}\right) \\
M_{\mathrm{TS}}^{l=1}\left(h_{0}=0.6\right)=m\left(\frac{\pi v}{e}+6.41655 \hbar\right)+o\left(\hbar^{2}\right), & M_{\mathrm{TS}}^{l=1}\left(h_{0}=0.9\right)=m\left(\frac{\pi v}{e}+60.9433 \hbar\right)+o\left(\hbar^{2}\right),
\end{array}
$$


as compared with the one-loop mass of the embedded Nielsen-Olesen vortex:

$$
M_{\mathrm{TS}}^{l=1}\left(h_{0}=0.0\right)=m\left(\frac{\pi v}{e}-1.67989 \hbar\right)+o\left(\hbar^{2}\right)
$$

Our numerical results suggest a breaking of the classical degeneracy, the NO vortices remaining as the ground states of the topological sector with $l=1$. These results are reinforced by the following qualitative argument. The long-distance behavior of the Seeley densities is:

1. Embedded ANO vortex $h_{0}=0.0: 2 \pi r \operatorname{tr} c_{1}^{I}(r) \propto \frac{1}{r}, 2 \pi r \operatorname{tr} c_{1}^{O}(r) \propto \frac{1}{r}, 2 \pi r \operatorname{tr} c_{2}^{I}(r) \simeq \mathcal{O}\left(\frac{1}{r^{3}}\right), 2 \pi r \operatorname{tr} c_{2}^{O}(r) \simeq$ $\mathcal{O}\left(\frac{1}{r^{3}}\right), 2 \pi r \operatorname{tr} c_{n}^{I}(r) \simeq \mathcal{O}\left(e^{-c r}\right), 2 \pi r \operatorname{tr} c_{n}^{O}(r) \simeq \mathcal{O}\left(e^{-c r}\right), n>2$, when $r \rightarrow \infty$.

2. Semi-local topological soliton $h_{0}>0.0: 2 \pi r \operatorname{tr} c_{n}^{I}(r) \propto \frac{1}{r}, 2 \pi r \operatorname{tr} c_{n}^{O}(r) \propto \frac{1}{r}, \forall n$, when $r \rightarrow \infty$.

If $h_{0}=0$, only the $c_{1}$ coefficient diverges, like $\log R$, but its contribution is cancelled by mass renormalization counter-terms. If $h_{0}>0$, all the Seeley coefficients are logarithmically divergent and infrared divergences grow out of control.

\section{References}

[1] T. Vachaspati, and A. Achucarro, Phys. Rep. 327 (2000) 347 .

[2] G.W. Gibbons, M.E. Ortiz, F. Ruiz Ruiz and T.M. Samols, Nucl.Phys. B385 (1992) 127 .

[3] A.A. Izquierdo, J.M. Guilarte, M.A.G. Leon, W. G. Fuertes, Nucl. Phys. B 635 (2002) 525-557, Nucl. Phys. B 638 (2002)378-404, Nucl. Phys. B 681 (2004) 163-194.

[4] M.Bordag, A.S. Goldhaber, P. van Nieuwenhuizen and D. Vassilevich, Phys. Rev. D 66 (2002) 125014

[5] D. Vassilevich, Phys. Rev. D 68 (2003) 045005 .

[6] A. Rebhan, P. van Nieuwenhuizen and R. Wimmer, Nucl. Phys. B 679 (2004) 382 .

[7] A.A. Izquierdo, J.M. Guilarte, M.T. Mayado and W. G. Fuertes, Phys. Rev. D70 (2004) 061702, Phys. Rev. D71 (2005) 125010, Jour. Phys. A39 (2006)6463-6471 .

[8] A.A. Izquierdo, J.M. Guilarte, M.T. Mayado and W. G. Fuertes, One-loop corrections to the mass of selfdual semi-local planar topological solitons, Nucl. Phys. B (2007), doi:10.1016/j.nuclphysb.2007.11.023, arXiv: 0707.4592 [hep-th].

[9] A.A. Izquierdo, J.M.M. Castañeda, J.M. Guilarte, M.A.G. Leon, M.T. Mayado, and W.G. Fuertes, Lectures on the mass of topological solitons, hep-th/0611180.

[10] M. Bordag, and I. Drozdov, Phys. Rev. D68(2003)065026 .

[11] H. Weigel, J. Phys. A39(2006)6799-6806 .

[12] N. Graham, V. Khemani, M. Quandt, O. Schroeder, and H. Weigel, Nucl. Phys. B707(2005) 233-277.

[13] N. Graham, M. Quandt, O. Schroeder, and H. Weigel, Nucl. Phys. B758(2006)112-143 .

[14] E. Elizalde, S. Odintsov, A. Romeo, A. Bytsenko, and S. Zerbini, Zeta regularization techniques with applications, World Scientific, Singapore, 1994.

[15] M. Bordag, E. Elizalde, K. Kirsten, and S. Leseduarte, Phys. Rev D56(1997)4896 . 